\title{
Computational Management Science Special Issue on "Optimisation methods and applications in the energy sector"
}

\author{
Stein-Erik Fleten ${ }^{1}$ - Daniel Kuhn ${ }^{2}$. \\ Afzal Siddiqui ${ }^{3,4}$
}

Historically state regulated, many industries within the energy sector, e.g., electricity and natural gas, have been gradually liberalised over the past three decades in an effort to improve economic efficiency (Wilson 2002). Instead of a vertically integrated paradigm with regulated monopolies that controlled both production and retail interests, decision makers within the energy sector must now cope with uncertain prices and competition from rivals. Exposure to such market forces was thought to encourage firms and consumers to foster technological innovation and to undertake efficiency investments, respectively. However, the transition towards a deregulated energy sector also meant that conventional, single-agent models for supporting investment and operational decisions may no longer be adequate. Indeed, in a survey of modelling tools for the electric power industry, Hobbs (1995) foresaw the need for a better representation of uncertainty and strategic interactions.

Along with deregulation, decarbonisation has more recently begun to transform the energy sector. In an attempt to mitigate the impact of climate change, policymakers in most industrialised countries have passed legislation to support investment in renewable energy technologies as well as to facilitate greater flexibility on the demand side

$凶 \quad$ Afzal Siddiqui

afzal.siddiqui@ucl.ac.uk

Stein-Erik Fleten

stein-erik.fleten@iot.ntnu.no

Daniel Kuhn

daniel.kuhn@epfl.ch

1 Norwegian University of Science and Technology, Trondheim, Norway

2 École Polytechnique Fédérale de Lausanne (EPFL), Lausanne, Switzerland

3 University College London, London, UK

4 Stockholm University, Stockholm, Sweden 
in the form of the so-called smart grid. Yet, these proposals also create new sources of risk and competition in the form of resource intermittency and markets for renewable energy certificates, respectively. Thus, both policymakers and participants in the energy sector would benefit from the development and application of appropriate optimisation methods in order to gain deeper insights into market design and operations.

In this special issue, we have five papers that address distinct aspects of the challenges posed by these twin trends of decarbonisation and deregulation in the energy sector. First, tackling the issue of demand-side flexibility in the form of energy storage coupled with renewable distributed generation output, "The impact of wind uncertainty on the strategic valuation of distributed electricity storage" by Pedro Crespo Del Granado, Stein W. Wallace, and Zhan Pang uses a stochastic programming approach to determine the value of a battery Del Granado et al. (2015). The authors use data from a UK site in order to demonstrate that considering uncertainty in wind output may increase the expected value of the storage system significantly compared to using a deterministic model.

Stochastic unit commitment problems become excruciatingly hard when many scenarios are incorporated. In order to ease the computational burden, Yonghan Feng and Sarah M. Ryan in "Solution sensitivity-based scenario reduction for stochastic unit commitment" devise, implement, and evaluate a scenario-reduction method, which selects a subset of all available scenarios that is small enough to guarantee the efficient and reliable solvability of the underlying unit commitment problem Feng and Ryan (2014). Based on real-world data for an Independent System Operator in the US, they demonstrate the practical applicability of their novel method, which is shown to produce reliable commitment schedules that compare favourably against the schedules obtained via the state-of-the-art methods in stochastic programming.

Due to the ongoing liberalisation, electricity prices in markets with price-setting gasfired power plants are increasingly correlated with natural gas prices. This phenomenon can be exploited for hedging. In "The natural hedge of a gas-fired power plant," Xiaojia Guo, Alexandros Beskos, and Afzal Siddiqui develop an insightful stochastic programming model that allows them to determine optimal hedging and generation decisions for a gas-fired power plant Guo et al. (2014). Based on UK energy price data, they find that producers benefit from hedging even though a perfect hedge is not available. It is also demonstrated that greater operational flexibility enhances the benefits of hedging, while higher energy-conversion efficiency has a detrimental effect.

From a system-wide perspective, expanding the transmission network is critical to facilitating investment in new generation plants. However, in most deregulated industries, transmission investment is typically undertaken by a cost-minimising transmission planner, whereas generation plant investment is handled by profit-maximising power companies. Via a game-theoretic model, "A leader-followers model of power transmission capacity expansion in a market driven environment" by Paolo Pisciella, Marida Bertocchi, and Maria Teresa Vespucci is able to address the effects of these conflicting objectives Pisciella et al. (2014). In particular, a novelty of their work is the exploration of transmission investment proposals as more emphasis is placed on reducing network congestion costs.

Finally, cost reductions and changes in electricity markets motivate deployment of energy storage capacity as well as transformer units into distribution grids. In 
"A dynamic programming model of energy storage and transformer deployments to relieve distribution constraints," Xiaomin Xi and Ramteen Sioshansi adopt an approximate dynamic programming approach and study a case of distribution grid deployment of storage and transformers under uncertainty of battery costs, imbalance sizes and prices, loads, and energy prices Xi and Sioshansi (2014). Data are taken from a utility in Ohio. Uses of storage include energy and ancillary service sales, backup supply, and more efficient use of distribution capacities such as transformers.

The papers in this special issue showcase the existing state-of-the-art modelling tools available for decision support. With the ongoing decarbonisation of the energy system, it is likely that further linkages between its components, e.g., due to storage and combined heat and power applications, will necessitate the development of more sophisticated methods for analysis. In turn, decision makers in both government and industry will be able to gain better insights about crafting policy and investment or operations, respectively. Indeed, for a complex system such as energy, the introduction of new technologies and sources of uncertainty will create more dependencies between consumption and production both spatially and temporally. Hence, modifying existing market designs or overhauling them altogether while anticipating the economic incentives of decision makers will become crucial to meeting the decarbonisation challenge successfully.

\section{References}

Del Granado PC, Stein WW, Zhan P (2015) The impact of wind uncertainty on the strategic valuation of distributed electricity storage. Comput Manag Sci. doi:10.1007/s10287-015-0235-0

Feng Y, Ryan SM (2014) Solution sensitivity-based scenario reduction for stochastic unit commitment. Comput Manag Sci. doi:10.1007/s10287-014-0220-z

Guo X, Beskos A, Siddiqui A (2014) The natural hedge of a gas-fired power plant. Comput Manag Sci. doi:10.1007/s10287-014-0222-X

Hobbs BF (1995) Optimization methods for electric utility resource planning. Eur J Oper Res 83(1):1-20

Pisciella P, Bertocchi M, Vespucci MT (2014) A leader-followers model of power transmission capacity expansion in a market driven environment. Comput Manag Sci. doi:10.1007/s10287-014-0223-9

Wilson RB (2002) Architecture of power markets. Econometrica 70(4):1299-1340

Xi X, Sioshansi R (2014) A dynamic programming model of energy storage and transformer deployments to relieve distribution constraints. Comput Manag Sci. doi:10.1007/s10287-014-0218-6 